\title{
Lqr Based Load Frequency Control By Introducing Demand Response
}

\author{
P.Venkatesh \\ Department of Electrical and Electronics Engineering, \\ V.R Siddhartha Engineering College, Vijayawada, AP, 520007, India \\ K.Srikanth \\ Department of Electrical and Electronics Engineering, \\ V.R Siddhartha Engineering College, Vijayawada, AP, 520007, India \\ V.Hari Vamsi \\ Department of Electrical and Electronics Engineering, \\ V.R Siddhartha Engineering College, Vijayawada, AP, 520007, India
}

\begin{abstract}
The strong inspiration for raise of renewable energy in power system, mainly at the demand side, results in novel changes in frequency and voltage regulation. In power system operation, wide area monitoring applications will focus heavily on Demand Response (DR), particularly in the smart grid area, where two-way communication and consumer sharing are involved. Load Frequency Control (LFC) model plays a significant role in electric power system design and operation. This paper presents an idea of incorporating a DR loop in conventional LFC known as LFC-DR, for a singlearea power system using Intelligent Controller. In the controller design DR communication delay latency is considered which linearized using Pade approximation. The DR control loop in LFC improves stability of the overall closed-loop system, efficiently increases the dynamic performance of the system. The simulation results show that the LQR controller based proposed power system model (LFC-DR) for single-area gives better performance than the traditional controller under any operating scenarios.
\end{abstract}

Index Terms - Demand Response (DR), Load Frequency Control (LFC), Linear Quadratic Regulator (LQR), Pade approximation, Stability

\section{INTRODUCTION}

Conventionally, frequency parameter in power system is maintained by balancing generation and demand via load i.e., spinning and non-spinning reserves [1]. Now a day's power generation, is mainly focus on renewable energy (RE) which are highly changeable. In this case, storage devices and responsive loads show huge pledge for balancing generation and demand, as they assist to neglect the usage of the common generation schemes, which are costly and/or environmentally distant. From the literature study it is known that, the idea of LFC model has determined only on the generation part, DR has not explained anywhere. The idea of the paper is to change common small-signal model of system utilized in LFC studies by incorporating DR control loop in traditional LFC (known as LFC-DR).

Demand response (DR) plays a significant role in power market by adjusting load rather than adjusting at generation side, to maintain balance between supply and demand. By the latest, smart grid technologies higher the integration of DR is achieved.

Other ideas of the paper is to construct the model as general as potential, to incorporate communication latency coupled with DR between the load aggregator companies (Lagcos) and the end-user customer devices. DR option is selected by the system operators based on the real-time electricity price.

\section{SYSTEM MODEL}

For frequency control analysis, generalized small-order linearized power system model is given by the power balance equation in the frequency-domain [5], [8]:

$$
\Delta P_{T}(s)-\Delta P_{L}(s)=2 H . s . \Delta f(s)+D . \Delta f(s)
$$

Where

$$
\Delta P_{T}(s)-\Delta P_{L}(s) \quad \text { Incremental power mismatch }
$$




$\begin{array}{ll}\Delta f(s) & \text { Frequency deviation } \\ 2 H & \text { Equivalent inertia constant } \\ \mathrm{D} & \text { Load damping coefficient } \\ \mathrm{S} & \text { Laplace transform operator }\end{array}$

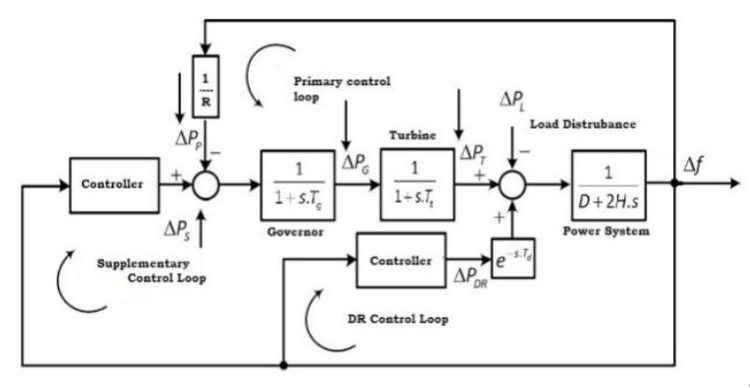

Fig.1. Proposed model of single area power system

The customized block diagram for single area power system (Thermal) with Demand Response (DR) control loop for LFC with communication latency is shown in Fig.1. DR presents spinning reserve in magnitude and power flow path i.e., if frequency difference becomes negative (positive), it is necessary to turn OFF (ON) a section of responsive loads for ancillary services, Equation (1) is changes as:

$$
\Delta P_{T}(s)-\Delta P_{L}(s)+\Delta P_{D R}(s)=2 H . s . \Delta f(s)+D . \Delta f(s)
$$

The power utilization of controllable loads can be varied immediately by the demand signal they accept. The only difficulty for DR is communication delay, identified as latency, which affects the dynamic behavior of the system. There are different methods fort linearization various problem with delays. In order to linearize the communication delay, Pade approximation is used in this study which is explained in the section IV.

\section{STATE-SPACE DYNAMICAL MODEL OF LFC-DR}

State-space representation is a helpful method for the purpose of the modern/robust control theory. For designing a common structure of LFC in dynamic frequency analysis this kind of representation can be easily changed and applied to any size of power system. The state-space model for LFC-DR is represented to show the impact of DR in LFC and controller design.

The state-space representation of proposed model (shown in Fig.1) is known by,

$$
\begin{gathered}
\dot{\mathrm{X}}(t)=A . \mathrm{X}(t)+B . u(t)+\Gamma \cdot \omega(t) \\
y(t)=C \cdot \mathrm{X}(t)
\end{gathered}
$$

Where

$$
\begin{array}{ll}
\text { A - System matrix, } & \mathrm{C}-\text { Observation matrix } \\
\mathrm{B}-\text { Control input matrix } & \Gamma \text {-Disturbance matrix } \\
\omega(t) \text { - Disturbance variable } & u(t) \text { - Input vector } \\
\Gamma-\text { Disturbance matrix } & y(t) \text {-System output } \\
\text { X - State vector } &
\end{array}
$$

To obtain the state-space model of the system, it is necessary to have the linear system. From Fig. 1 it is observed that the model as a non-linear element that is time delay proposed model. The time delay is linearized by the approximation known as Pade approximation.

\section{PADE APPROXIMATION}

In turn, to linearize the time delays in control engineering the Pade approximation is commonly used. Pade approximation is one of the frequently used methods to approximate a dead-time by rational functions, but with the numerator and denominator with same degrees is most widely suggested. [7]

The pade function for the time delay is defined by,

$$
e^{-s \cdot T_{d}} \approx R_{p q}\left(-s . T_{d}\right)
$$


It is as follows:

$$
R_{p q}\left(e^{-s . T_{d}}\right)=D_{p q}\left(e^{-s . T_{d}}\right)^{-1} \cdot N_{p q}\left(e^{-s . T_{d}}\right)
$$

Where

$$
\begin{aligned}
& N_{p q}\left(e^{-s . T_{d}}\right)=\sum_{k=0}^{p} \frac{(p+q-k) ! p !}{(p+q) ! k !(p-q) !} \cdot\left(-s \cdot T_{d}\right)^{k} \\
& D_{p q}\left(e^{-s . T_{d}}\right)=\sum_{k=0}^{q} \frac{(p+q-k) ! q !}{(p+q) ! k !(q-k) !} \cdot\left(-s \cdot T_{d}\right)^{k}
\end{aligned}
$$

' $N_{p q}$ ' and ' $D_{p q}$ ' are the polynomials of order 'p' and 'q' respectively. It is common to use same order of numerator and denominator for the approximation and the order generally varies between 1 to 10 . In this study $5^{\text {th }}$ order Pade approximation is used, because the low-pass filters i.e., speed-governor and turbine, in the model is less than $15 \mathrm{rad} / \mathrm{sec}$.

\section{a) Steady-State Error:}

\section{ANALYTICAL EVALUATION OF THE MODEL}

The primary control loop in Fig. 1 is the fastest proposed control action in a system but it is not adequate to make the frequency deviation to zero at steady-state. To overcome these, the supplementary frequency control loop shown in Fig.1. By adding DR, it is essential to examine the effect of DR loop on the steady-state error shown in Fig.1. The optimal distribution between DR and supplementary control loops is calculated from the steady state error estimation for controller design. The equations of conventional LFC at steady-state are well recognized from [8]. By DR control loop the system frequency deviation can be expressed as follows:

$$
\Delta f(s)=\frac{1}{2 H . s+D}\left[\Delta P_{T}(s)-\Delta P_{L}(s)+G(s) \cdot \Delta P_{D R}(s)\right]
$$

Where,

$$
\begin{aligned}
& \Delta P_{T}(s)=H(s) \cdot\left[\Delta P_{S}(s)-\frac{1}{R} \cdot \Delta f(s)\right] \\
& H(s)=\frac{1}{\left(1+s \cdot T_{g}\right)\left(1+s \cdot T_{t}\right)} \\
& G(s)=\frac{-s^{5}+\frac{30}{T} s_{d}^{4}-\frac{420}{T_{d}^{2}} \cdot s^{3}+\frac{3360}{T_{d}^{3}} \cdot s^{2}-\frac{15120}{T_{d}^{4}} \cdot s+\frac{30240}{T_{d}^{5}}}{s^{5}+\frac{30}{T_{d}} \cdot s^{4}+\frac{420}{T_{d}^{2}} \cdot s^{3}+\frac{3360}{T_{d}^{3}} \cdot s^{2}+\frac{15120}{T_{d}^{4}} \cdot s+\frac{30240}{T_{d}^{5}}}
\end{aligned}
$$

Based on the final value theorem, the steady-state frequency deviation can be obtained as follows [8].

$$
\Delta f_{S S}=\frac{\Delta P_{S, S S}+\Delta P_{D R, S S}-\Delta P_{L}}{D+\frac{1}{R}}
$$

From equation (11) the following conclusions can be observed

- Not dependent on the delay and the approximation order.

- The high reliability of frequency regulation can be achieved with DR.

- The control effort is split between supplementary and

DR loops for zero frequency deviation at steady-state.

By taking above conclusions into account: with DR in the LFC, the control effort can be shared as follows based on cost of electricity in the real market.

$$
\begin{aligned}
& \Delta P_{S}(s)=\alpha . \text { Control effort } \\
& \Delta P_{D R}(s)=(1-\alpha) . \text { Control effort }
\end{aligned}
$$

On by splitting control effort between two loops the system is modified and is governed by the,

$$
(1-\alpha) \cdot G(s)+\alpha \cdot H(s)
$$

Where $\alpha$ varies between 0 to 1 . For alpha $=1$, the total reserves is provided by usual regulation services. For alpha $=0$, the total control is guaranteed by DR. The alpha value is determined by ISO/RTO, depend on the cost of DR and usual regulatory 
services in the real time market [2]. The simulation studies are passed with two different values of alpha for frequency deviation on the system.

\section{b) Sensitivity Analysis with and without DR:}

A systematic way is used to know the effect of DR loop on the overall sensitivity of the closed-loop system w.r.t the openLoop system. Second analysis is the sensitivity of the closed-loop system w.r.t alpha. For this analysis Fig.1 is slightly changed with single integral controller (with gain K) for both control loops shown in Fig.2. This change permit to share the necessary control effort between two loops as discuss earlier.

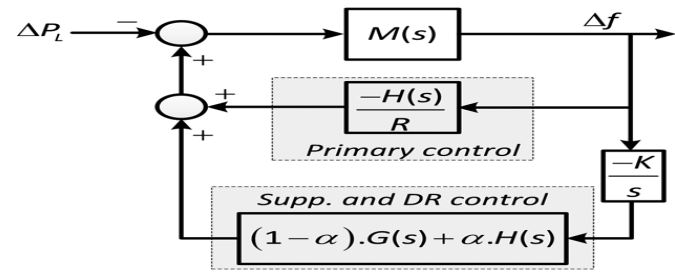

Fig.2 Proposed system with integral controller.

The sensitivity analysis of the closed-loop system w.r.t the open-loop system, for the system with and without DR is written as follows [8],

$$
\begin{aligned}
S_{O L}^{D R} & =\frac{\frac{\partial T_{D R}}{\partial T_{O L}}}{\frac{T_{D R}}{T_{O L}}}=\frac{\frac{\partial T_{S}}{\partial T_{O L}}}{S_{O L}^{S}=}=\frac{\left(T_{O L}(s)\right)^{-1}-\frac{K}{s} \cdot \alpha \cdot H(s)-\frac{K}{s} \cdot(1-\alpha) \cdot G(s)}{\frac{T_{S}}{T_{O L}}}=\frac{\left(T_{O L}(s)\right)^{-1}}{\left(T_{O L}(s)\right)^{-1}-\frac{K}{s} \cdot H(s)}
\end{aligned}
$$

From equation (15) the relation of sensitivity function can be derived as,

$\frac{S_{O L}^{D R}}{S_{O L}^{S}}=\frac{\left(T_{O L}(s)\right)^{-1}-\frac{K}{s} \cdot H(s)}{\left(T_{O L}(s)\right)^{-1}-\frac{K}{s} \cdot \alpha \cdot H(s)-\frac{K}{s}(1-\alpha) \cdot G(s)}$

As the alpha also plays an important role in the LFC-DR it is essential to check the sensitivity function of the closed-loop system w.r.t alpha. The sensitivity function w.r.t to alpha is given by,

$$
S_{\alpha}^{D R}=\frac{\frac{\partial T_{D R}}{\partial \alpha}}{\frac{T_{D R}}{\alpha}}=\frac{\alpha \cdot \frac{K}{s}(H(s)-G(s))}{\left(T_{O L}(s)\right)^{-1}-\frac{K}{s} \cdot \alpha \cdot H(s)-\frac{K}{s} \cdot(1-\alpha) \cdot G(s)}
$$

The simulation results are compared with different alpha values and are discussed in section VII

\section{c) Stability Analysis with and without DR:}

In control systems stability plays an important role. In this section, the gain and phase margins for two different values of alpha are explained. The open loop and closed loop transfer function with load disturbance is given by,

$$
\begin{gathered}
1+\underbrace{\frac{H(s) \cdot M(s)}{R}+\frac{K}{s} \cdot \alpha \cdot H(s) \cdot M(s)+\frac{K}{s} \cdot(1-\alpha) \cdot G(s) \cdot M(s)}_{v^{D R}}=0 \\
1+\underbrace{\frac{H(s) \cdot M(s)}{R}+\frac{K}{s} \cdot H(s) \cdot M(s)}_{v^{s}}=0
\end{gathered}
$$

The simulation results are compared with different alpha values and are discussed in the section VII

\section{CONTROLLER DESIGN}

Various modern control theories have been used for the LFC model. The general controller design (LQR) method for the 
LFC-DR model is presented in this section. The optimal controller "Linear- Quadratic regulator (LQR)" theory deals with minimizing cost function of a dynamic system. The Optimal control in state-space is related with Riccati Equation with state variable functions which are solved by the control law.

The basic design of the LQR controller is to minimized the performance index of the system given in equation (3)

$$
\left.\mathfrak{I}=\int_{0}^{\infty} x^{T} \cdot Q \cdot x+\rho \cdot u^{T} \cdot R \cdot u\right] \cdot d t
$$

Where, $\rho$ is weighting factor chosen by designer

$\mathrm{Q}$ is $n \times n$ semi definite symmetric state cost matrix

$\mathrm{R}$ is $m \times m$ positive definite symmetric control cost matrix

$x^{T}=\left[\Delta f, \Delta P_{g}, \Delta P_{t}, x_{4}, x_{5}, x_{6}, x_{7}, x_{8}\right]$

$x_{4}, x_{5}, x_{6}, x_{7}, x_{8}$ are the states associated with pade

Approximation

From equation (12) \& (13) the control input to the supplementary and DR controller is given by,

$$
u_{1}=\frac{\alpha}{1-\alpha} \cdot u_{2} \quad \text { (Or) } \quad u_{2}=\frac{1-\alpha}{\alpha} \cdot u_{1}
$$

The two inputs are unified as single input, all the matrices remain same except B matrix in state-space, the modified B matrix by including unified control input $u_{2}=F\left(u_{1}\right)$ is given by,

$$
B^{T}=\left[\begin{array}{llllllll}
\frac{\alpha-1}{2 H . \alpha} & 0 & \frac{1}{T_{g}} & \frac{16(1-\alpha)}{\alpha} & 0 & 0 & 0 & 0
\end{array}\right]
$$

The simulation results are carried out for two cases of unified inputs which are shown in Fig.7

To make zero steady-state error for frequency deviation, the traditional full-state feedback cannot achieve. So, integral control is required to overcome the above problem. With the modified state space equations ensuring the system matrix is controllable [9].

$$
\begin{aligned}
{\left[\begin{array}{c}
\dot{\mathrm{X}} \\
\dot{x_{I}}
\end{array}\right] } & =\left[\begin{array}{ll}
A & 0 \\
C & 0
\end{array}\right] \cdot\left[\begin{array}{c}
\mathrm{X} \\
x_{I}
\end{array}\right]+\left[\begin{array}{c}
\hat{B} \\
0
\end{array}\right] \cdot \hat{u}+\left[\begin{array}{c}
\Gamma \\
0
\end{array}\right] . \omega \\
y & =\left[\begin{array}{ll}
C & 0
\end{array}\right] \cdot\left[\begin{array}{c}
\mathrm{X} \\
x_{I}
\end{array}\right]
\end{aligned}
$$

The control law for state feedback is defined only if the system matrix is controllable, given by

$$
\hat{u}=-\left[\begin{array}{ll}
K & K_{1}
\end{array}\right] \cdot\left[\begin{array}{l}
\mathrm{X} \\
x_{1}
\end{array}\right]=-\tilde{K} \cdot \tilde{\mathrm{X}}
$$

To use the LQR method, $\mathrm{Q}$ and $\mathrm{R}$ are the state and control weighting matrices (scalar quantities) to be known. Q and $\mathrm{R}$ selected based on the frequency response requirements.

In this study, MATLAB command is used for LQR implementation. "K = lqr $[A, B, Q, R]$ "for finding the feedback gain value.

\section{V11. SIMUATION RESULTS}

The parameters utilized in the simulation studies for the proposed model (LFC-DR) for single-area is given in Table. I

TABLE.I

PARAMETERS FOR THE SIMULATION STUDY

\begin{tabular}{|l|c|c|l|l|l|l|l|}
\hline$T_{g}$ & $T_{t}$ & $\mathrm{R}$ & $2 \mathrm{H}$ & $\mathrm{D}$ & $T_{d}$ & $\Delta P_{L}$ & $\mathrm{~K}$ \\
\hline $\begin{array}{l}0.08 \\
\text { sec }\end{array}$ & $\begin{array}{c}0.4 \\
\mathrm{sec}\end{array}$ & $\begin{array}{c}3.0 \\
\mathrm{~Hz} / \mathrm{p} . \mathrm{u}\end{array}$ & $\begin{array}{l}0.1667 \\
\text { pu.sec }\end{array}$ & 0.015 & 0.1 & 0.01 & 0.2 \\
& & & & & $\mathrm{sec}$ & $\mathrm{sec}$ & \\
\hline
\end{tabular}

Fig. 3 shows the effect of DR loop for two different values of alpha. For alpha=0.1, the system is less sensitive to the closed-loop system w.r.t the open-loop system, i.e., $10 \%$ from traditional regulation and $90 \%$ from DR resources. When, alpha $=0.8$, the 
system is highly sensitive to the closed-loop system w.r.t the open-loop system, here $80 \%$ if from traditional reserves and $20 \%$ from DR reserves.

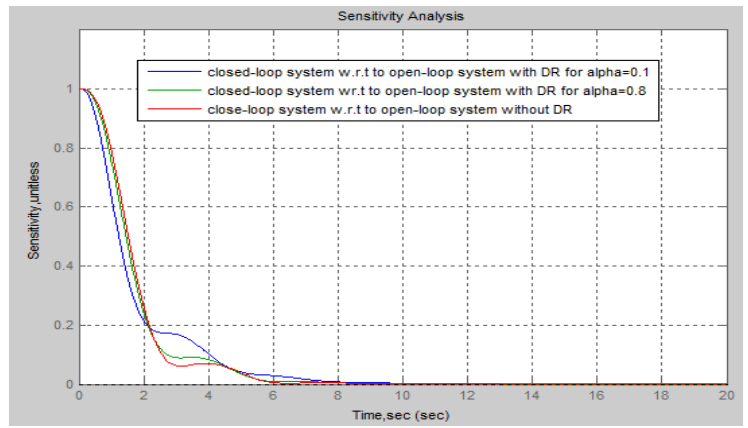

Fig.3 Sensitivity of closed -loop system w.r.t to open-loop system

Fig.4 shows the Sensitivity analysis of closed-loop system w.r.t the alpha . For alpha $=0.1$, the closed-loop system is less sensitive to alpha, the higher share in frequency regulation is provided by DR control loop.

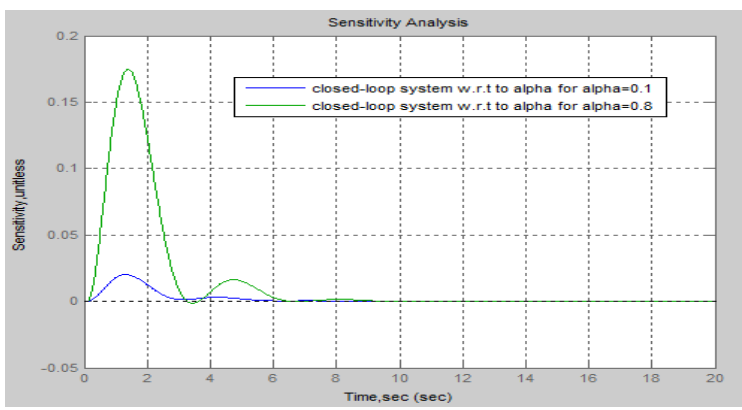

Fig.4 Sensitivity of closed-loop system w.r.t alpha

Bode plot of the system with and without DR is shown in Fig.5. The gain and phase margins is shown in Table. II. It is observed that smaller the alpha value (alpha $=0.1$ ) higher the gain and phase margin is obtained, results in stable system.

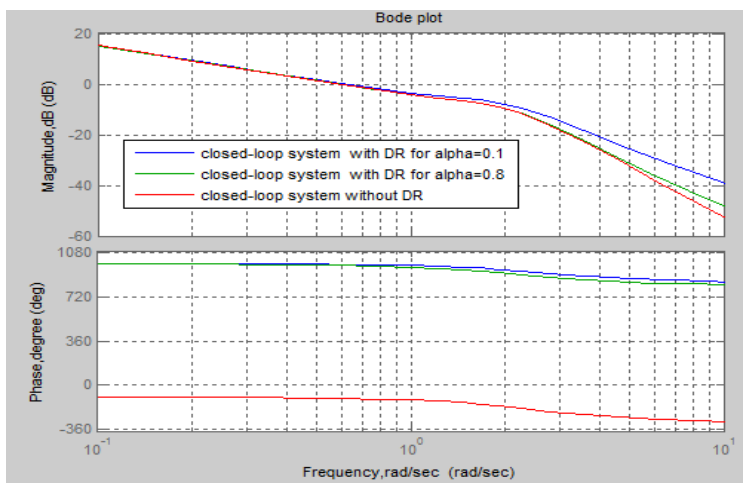

Fig. 5 Bode plot for LFC with and without DR

TABLE. II

PHASE AND GAIN MARGINS FOR SYSTEM (LFC) WITH AND WITHOUT DR

\begin{tabular}{|c|c|c|c|}
\hline & $\begin{array}{c}\text { Closed-loop } \\
\text { system for } \\
\text { alpha=0.1 }\end{array}$ & $\begin{array}{c}\text { Closed-loop } \\
\text { system for } \\
\text { alpha=0.8 }\end{array}$ & $\begin{array}{c}\text { Closed-loop } \\
\text { system } \\
\text { without DR }\end{array}$ \\
\hline $\begin{array}{c}\text { Gain margin, } \\
\text { dB }\end{array}$ & 14.1 & 11.3 & 10.3 \\
\hline $\begin{array}{c}\text { Phasemargin, } \\
\text { degree }\end{array}$ & 83.5 & 74.8 & 72.2 \\
\hline
\end{tabular}


The simulation was carried out in MATLAB/ Control system toolbox for the proposed system for significant explanation. For the comparison, LQR design is employed is used for both systems with and without to show the effectiveness of the system. In the first simulation study, 0.01 p.u load disturbance is applied for the traditional LFC and LFC-DR. It is observed, when alpha $=0.1$ it is noticed that LFC-DR model gives superior performance than the traditional LFC throughout transient period. The same process is repeated for alpha $=0.8$.

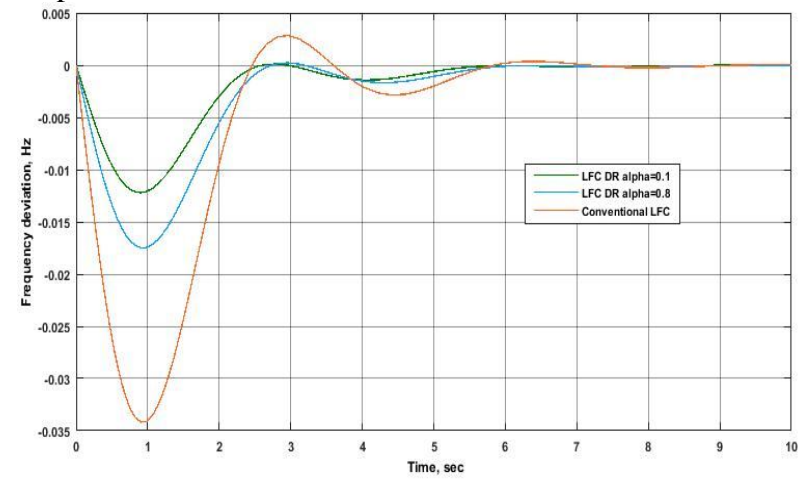

Fig.6 Frequency deviation for LFC and LFC-DR

As discussed in section.6, the control inputs are unified as the function of alpha. The unification is made in two ways: $u_{1}=F\left(u_{2}\right)$ or $u_{2}=F\left(u_{1}\right)$. To show the impact of unification simulation was carried out to evaluate the behavior of the system for both cases, which is shown in Fig.7. It is noticed that the variation between two unifying approaches is small.

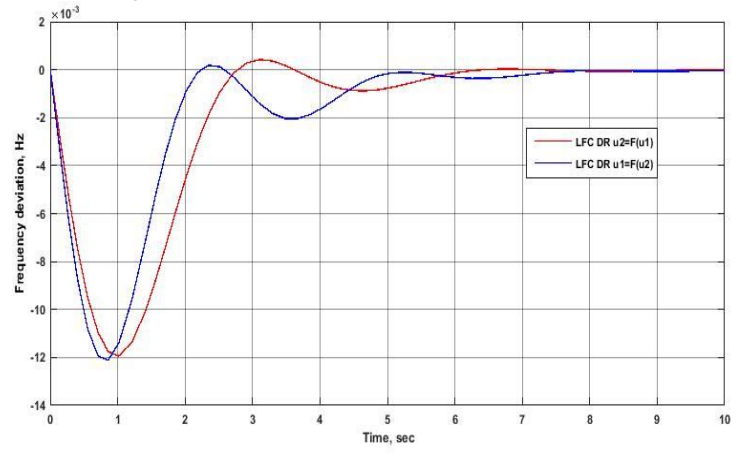

Fig.7 Unified inputs on LFC-DR

The communication delay in DR loop plays an important role in LFC-DR for frequency stabilization. The simulation is performed to know the impact of different values of latency for different values of alpha, shown in Fig.8

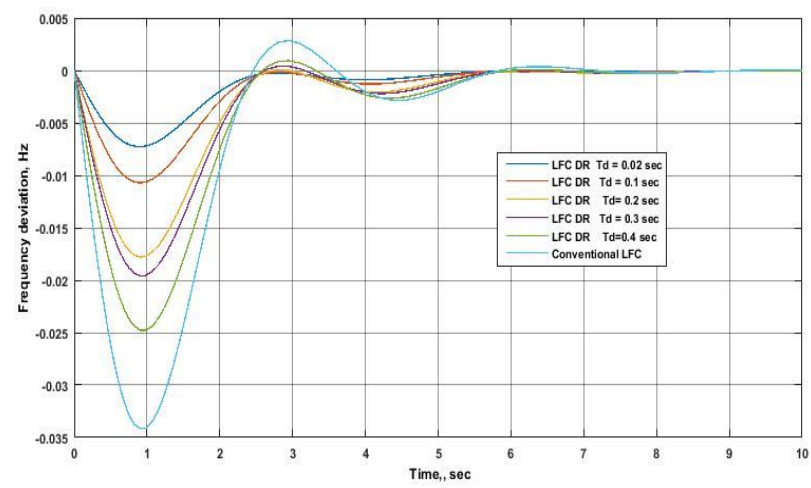

Fig.8 Impact of latency on the LFC-DR

The proposed model gives improved performance when compared to the traditional LFC when communication delay is less than $0.2 \mathrm{sec}$. With the time delay more than $0.2 \mathrm{sec}$ the response is most horrible than the conventional LFC. 
International Journal of Innovations in Engineering and Technology (IJIET)

http://dx.doi.org/10.21172/ijiet.83.026

\section{STEADY-STATE VALUES}

\begin{tabular}{|c|c|c|}
\hline Type of the system & $\begin{array}{c}\text { Supplementary } \\
\text { loop }\end{array}$ & DR loop \\
\hline LFC-DR for alpha $=0.8$ & 0.008 p.u & 0.002 p.u \\
\hline LFC-DR for alpha $=0.1$ & 0.001 p.u & 0.009 p.u \\
\hline
\end{tabular}

TABLE.IV

NUMERICAL ANALYSIS FOR CHANGE IN FREQUENCY

\begin{tabular}{|l|c|c|}
\hline \multicolumn{1}{|c|}{ Type of the system } & $\begin{array}{c}\text { Settling time } \\
(\mathrm{sec})\end{array}$ & $\begin{array}{c}\text { Undershoot } \\
(\mathrm{p} . \mathrm{u})\end{array}$ \\
\hline Conventional LFC & $>10$ & 0.0333 \\
\hline LFC-DR for alpha $=0.8$ & $>8$ & 0.018 \\
\hline LFC-DR for alpha $=0.1$ & 7 & 0.011 \\
\hline
\end{tabular}

From the above discussed simulation studies, it is observed that the proposed method gives better results and robust. It can also be practical and results are seen at different perturbations using different traditional controllers as well as the proposed controllers for LFC-DR model.

\section{CONCLUSION}

In this paper, LFC-DR problem is solved by using the optimal LQR controller. The DR communication latency is considered which is linearized by pade approximation. The settling time and undershoot values are considered to explain the robustness of the proposed controller.

\section{REFERENCE}

[1] R. Doherty and M. O'Malley, "A new approach to quantify reserve demand in systems with significant installed wind capacity ," IEEE Trans. Power Syst., vol. 20, no. 2, pp. 587-595, May 2005

[2] S.A.Pourmousavi and M.H.Nehrir, " Real-time central demand response for primary frequency regulation in microgrids,” IEEE Trans.Smart Grid, Vol.3, No.4,Dec.2012, pp.1988-1996.

[3] Mahdi Behrangrad, Hideharu Sugihara and Tsuyoshi Funaki, "Analysis of optimal spinning procurement with demand response resources under social cost minimization,” IEEJ Trans. Electrical and Electronic Enginnering, Vol.6, issue 3, May 2011, pp 211-220

[4] U.S Department of energy, Smart Grid, Sep 28, 2011 [online]

http://energy.gov/oe/technology-development/smart-grid

[5] P. Kundur, Power system stability and control. New York, NY, USA: McGraw-Hill, 1994, ch.11.

[6] Mohyi ed-dm Azzam, “ An optimal Approach to Robust Controller for Load-Frequency Control,’IEEE 2002

[7] G.H.Golub and C.F. Van Loan, Matrix Computations $3^{\text {rd }}$ edition Baltimore, MD, USA: Johns Hopkins Unversity, Press, 1996, pp.572-574

[8] H. Bevarani, Robust Power System Frequency Control, New York, NY, USA: Springer, 2009, ch.1-3

[9] R.C. Dorf and R.H.Bishop, Modern Control Systems, $7^{\text {th }}$ edition. New York, NY, USA: Addition-Wesley, 1995, pp.807

[10] S.Ali Pourmousavi, M.Hashem Nehir, “Introducing Dynamic Demand Response in the LFC model,” IEEE Trans. Power Sysy., Vol.29, No.4, July 2014, pp.1562-1572 